\title{
Early influenza vaccine effectiveness results 2015-16: I-MOVE multicentre case-control study
}

E Kissling ${ }^{1}$, M Valenciano $^{1}$

1. EpiConcept, Paris, France

Correspondence: Esther Kissling (e.kissling@epiconcept.fr)

Citation style for this article:

Kissling E, Valenciano M. Early influenza vaccine effectiveness results 2015-16: I-MOVE multicentre case-control study. Euro Surveill. 2016;21(6): pii=30134. D0I: http://dx.doi.org/10.2807/1560-7917.ES.2016.21.6.30134

Article published on 11 February 2016

On 11 February 2016, the Influenza Monitoring Vaccine Effectiveness in Europe (I-MOVE) published the 201516 interim vaccine effectiveness (VE) estimates against influenza from a multi-centre case control study in 10 study sites: Germany, France, Hungary, Ireland, Italy, Poland, Portugal, Spain, Sweden and the Netherlands, on their website [1].

Adjusted VE interim results against any influenza among all ages were at $46.3 \%$ (95\% confidence interval (Cl): $4.9-69.7 \%)$ and $45.2 \%(95 \% \mathrm{Cl}:-12.5-73.3 \%)$ among the 18-64 year olds. Among those aged 65 years and older, there were only 14 influenza cases in the study. The adjusted VE against influenza $A\left(\mathrm{H}_{1} \mathrm{~N}_{1}\right)$ pdmog was at $44.2 \%$ (95\% Cl: $-3.1-69.8 \%)$ among all ages and thus lower compared with end of season estimates published in previous years $(55.5 \%$ in $2010-11,50.4 \%$ in $2012-13 ; 47.5 \%$ in $2013-14,54.2 \%$ in 2014-15).

Early season influenza VE was measured against medically-attended laboratory-confirmed influenza from week 41/2015 to week 3/2016 using a test-negative design as described in the I-MOVE generic protocol [2] and in the I-MOVE multicentre case-control publications [3]. Some 1,933 influenza-like illness patients among whom 348 were positive to influenza were included: four cases of influenza A not subtyped, 246 $A\left(\mathrm{H}_{1} \mathrm{~N}_{1}\right) p d m o 9,21 \mathrm{~A}\left(\mathrm{H}_{3} \mathrm{~N}_{2}\right)$, and 77 influenza $B$ cases. Among the 37 influenza $B$ cases where lineage was available, 36 (97.3\%) were of the Victoria lineage, a lineage not included in the trivalent vaccine.

For this interim analysis, there was no information on genetic characterisation of the viruses. The recently published European Centre for Disease Prevention and Control risk assessment [4] reported that all $A\left(\mathrm{H}_{1} \mathrm{~N}_{1}\right)$ pdmog viruses characterised in the European Union up to week three belonged to the $6 \mathrm{~B}$ subgroup.
The interim estimates should be interpreted with caution. The 2015-16 season started late in the participating countries and the sample size for these interim estimates is low, resulting in low precision. The final estimates will be available at the end of the influenza season.

Read more here.

\section{References}

1. Influenza Monitoring Vaccine Effectiveness in Europe (I-MOVE). Interim 2015-16 influenza vaccine effectiveness results, I-MOVE multicentre case-control study. I-MOVE; 2016. Available from: https://sites.google.com/site/epiflu/ Home/2015-16-interim-results

2. European Centre for Disease Prevention and Control (ECDC). Protocol for case control studies to measure pandemic and seasonal vaccine effectiveness in the European Union and European Economic Area. Stockholm: ECDC; 2010. Available from: http://www.ecdc.europa.eu/en/publications/ Publications/0907_TED_Influenza_AH1N1_Measuring _ Influenza_Vaccine_Effectiveness_Protocol_Case_Control_ Studies.pdf

3. Influenza Monitoring Vaccine Effectiveness in Europe (I-MOVE). I-MOVE website list of publications 2007-2015. I-MOVE; 2016. Available from: https://sites.google.com/site/epiflu/ list-of-i-move-publications

4. European Centre for Disease Prevention and Control (ECDC). Risk Assessment: Seasonal influenza 2015-16 in the EU/EEA countries. Stockholm: ECDC; 2016. Available from: http://ecdc. europa.eu/en/publications/Publications/seasonal-influenzarisk-assessment-2015-2016.pdf

License and copyright

This is an open-access article distributed under the terms of the Creative Commons Attribution (CC BY 4.0) Licence. You may share and adapt the material, but must give appropriate credit to the source, provide a link to the licence, and indicate if changes were made.

This article is copyright of the authors, 2016. 